\title{
Effects of synbiotic food consumption on glycemic status and serum hs-CRP in pregnant women: a randomized controlled clinical trial
}

\author{
Mohsen Taghizadeh, Zatolla Asemi
}

Research Center for Biochemistry and Nutrition in Metabolic Diseases, Kashan University of Medical Sciences, Kashan, I.R. Iran

\begin{abstract}
OBJECTIVE: The aim of this study was to determine the effects of synbiotic food consumption on glycemic status and serum high sensitivity C-reactive protein (hs-CRP) levels of Iranian pregnant women. DESIGN: This randomized placebo-controlled clinical trial was performed among 52 pregnant women, primigravida, aged 18-35 year old, in their third trimester. After a 2-wk run-in period, subjects were randomly assigned to consume either a synbiotic $(n=26)$ or control food $(n=26)$ for 9 weeks. The synbiotic food consisted of a probiotic Lactobacillus sporogenes $\left(1 \times 10^{7} \mathrm{CFU}\right), 0.04 \mathrm{~g}$ inulin as prebiotic with $0.38 \mathrm{~g}$ isomalt, $0.36 \mathrm{~g}$ sorbitol and 0.05 g stevia as sweetener per $1 \mathrm{~g}$. Control food (the same substance without probiotic bacteria and inulin) was packed in identical 9-gram packages. Patients were asked to consume the synbiotic and control foods two times a day. Fasting blood samples were taken at baseline and after a 9-wk intervention for quantification of related factors. RESULTS: Consumption of a synbiotic food did not show any significant change regarding the impact of insulin actions in the synbiotic group; nonetheless, compared to the control food, it resulted in a significant decrease in serum insulin levels (-0.26 vs. $6.34 \mu \mathrm{IU} / \mathrm{mL}, \mathbf{P}=0.014)$ and HOMA-IR (-0.13 vs. 1.13, P=0.033), a significant difference in HOMA-B (5.30 vs. 34.22, $P=0.040)$ and a significant rise in QUICKI score (0.002 vs. $\mathbf{- 0 . 0 2}, \mathbf{P}=\mathbf{0 . 0 2 2})$. CONCLUSIONS: Consumption of a synbiotic food for 9 weeks by pregnant women had beneficial effects on insulin actions compared to the control food, but did not affect FPG and serum hs-CRP concentrations.
\end{abstract}

Key words: Glycemic status, High sensitivity C-reactive protein, Pregnant women, Synbiotic

\section{INTRODUCTION}

Insulin resistance is a physiological condition in

Address for correspondence:

Zatollah Asemi, PhD, Department of Biochemistry and

Nutrition, Kashan University of Medical Sciences, Iran,

Tel.: +98-361-4463378, Fax: +98-361-4463377,

E-mail: asemi_r@yahoo.com

Received 20-10-2013, Accepted 21-11-2013 which circulating levels of insulin are inadequate to elicit a metabolic response from adipose tissue, skeletal muscle, liver cells and other non-traditional insulinsensitive tissues. ${ }^{1}$ Pregnancy-induced production of cytokines, owing to excess body weight, ${ }^{2}$ including leptin, ${ }^{3}$ resistin, ${ }^{4}$ interleukin-6 (IL-6), ${ }^{5,6}$ together with low physical activity ${ }^{7}$ is associated with abnormal glucose homeostasis, insulin resistance and increased systemic 
inflammation, particularly in the third trimester. Maternal hyperinsulinism and glycemic status as well as increased inflammatory markers typically result in preterm delivery, ${ }^{8}$ higher rate of cesarean section, ${ }^{9}$ the development of pregnancy-induced hypertension (PIH) and gestational diabetes mellitus (GDM). ${ }^{10}$ GDM affects $1-14 \%$ of pregnancies depending on different screening methods, diagnostic criteria and the population screened. ${ }^{11}$ It is related to several adverse pregnancy outcomes, including shoulder dystocia, neonatal hypoglycemia, respiratory distress, hypocalcemia, ${ }^{12}$ macrosomia ${ }^{13}$ and increased risk of developing type 2 diabetes (T2D) later in life. ${ }^{14}$

The primary treatment for insulin resistance and inflammation is diet therapy, especially low-glycemic load diet, exercise ${ }^{15}$ and antioxidant supplementation including vitamins $\mathrm{E}$ and $\mathrm{C} .{ }^{16}$ In addition, use of antidiabetic medications ${ }^{17}$ and anti-inflammatory agents ${ }^{18}$ are suggested for decreased insulin resistance and inflammatory factors in pregnant women. Although several attempts have been made to decrease insulin resistance and inflammatory factors by consumption of probiotic-containing products among pregnant women, ${ }^{19,20}$ limited data are available assessing the effects of synbiotic foods. Furthermore, earlier studies on the effects of synbiotics have mostly been assessed in non-pregnant ${ }^{21}$ and animal models. ${ }^{22}$ Our previous study showed that synbiotic food consumption in diabetic patients led to decreased serum insulin and hs-CRP levels after 6 weeks. ${ }^{21}$ However, 3 months of synbiotic supplementation did not promote any significant changes in inflammatory cytokines among healthy elderly individuals. ${ }^{23}$

Synbiotics are thought to affect insulin resistance and inflammation by short-chain fatty acid (SCFA) production ${ }^{24,25}$ and decreased expression of inflammation-relevant genes, including interleukin-6 (IL-6), IL-8, cyclooxygenase-2 (COX2) and IL-1 $\alpha .^{6}$ To our knowledge, no reports are available indicating the favorable effects of synbiotic food consumption on glycemic status and inflammatory markers in pregnant women. The aim of the current study was, therefore, to investigate the effects of synbiotic food consumption on glycemic status and serum high sensitivity C-reactive protein (hs-CRP) levels among Iranian pregnant women.

\section{METHODS}

\section{Participants}

This randomized placebo-controlled clinical trial was performed in Kashan, Iran, from June 2012 to February 2013. To estimate the sample size, we used a randomized clinical study sample size formula where type one (a) and type two error (b) were 0.05 and 0.20 (power $=80 \%$ ), respectively. Based on a previous study, ${ }^{21}$ we also considered 6.5 as the difference in the mean (d) of insulin as a key variable. The formula showed that the current study needed 26 subjects per each group to achieve $80 \%$ of the power. Pregnant women, primigravida, aged $18-35$ years old who were carrying a singleton pregnancy at 27 weeks of gestation were included in this study. We excluded those with pre-eclampsia, hypertension, GDM, complete bed rest (CBR), hospitalization, intra-uterine fetal death (IUFD), intrauterine growth retardation (IUGR) as well as those with a history of rheumatoid arthritis, thyroid, parathyroid or adrenal diseases and hepatic or renal failure, smokers and those taking medications including nonsteroidal anti-inflammatory drugs (NSAIDs) and aspirin. Gestational age was assessed from the date of the last menstrual period and from clinical assessment. ${ }^{27}$ Individuals with the abovementioned inclusion criteria were invited to participate in the study from women attending maternity clinics affiliated to Kashan University of Medical Sciences, Kashan, Iran. A total of 86 pregnant women aged 18-35 years old were screened; of these, 56 pregnant women met the inclusion criteria. Participants were randomly assigned to receive synbiotic $(n=28)$ or control food $(n=28)$ for 9 weeks. Among the individuals in the synbiotic group, 2 persons [hospitalization $(n=1)$ and IUGR $(n=1)]$ were excluded. The exclusions in the control group were also 2 women [pre-eclampsia $(n=1)$ and GDM $(n=1)]$. Finally, 52 participants [synbiotic $(\mathrm{n}=26)$ and control food $(n=26)]$ completed the trial (Figure 1). The study was conducted according to the guidelines laid down in the Declaration of Helsinki. The ethical committee of Kashan University of Medical Sciences (KUMS) approved the study and informed written consent was obtained from all participants. The trial was registered in the Iranian website (www.irct.ir) for registration of clinical trials (IRCT code: IRCT201212105623N3). 


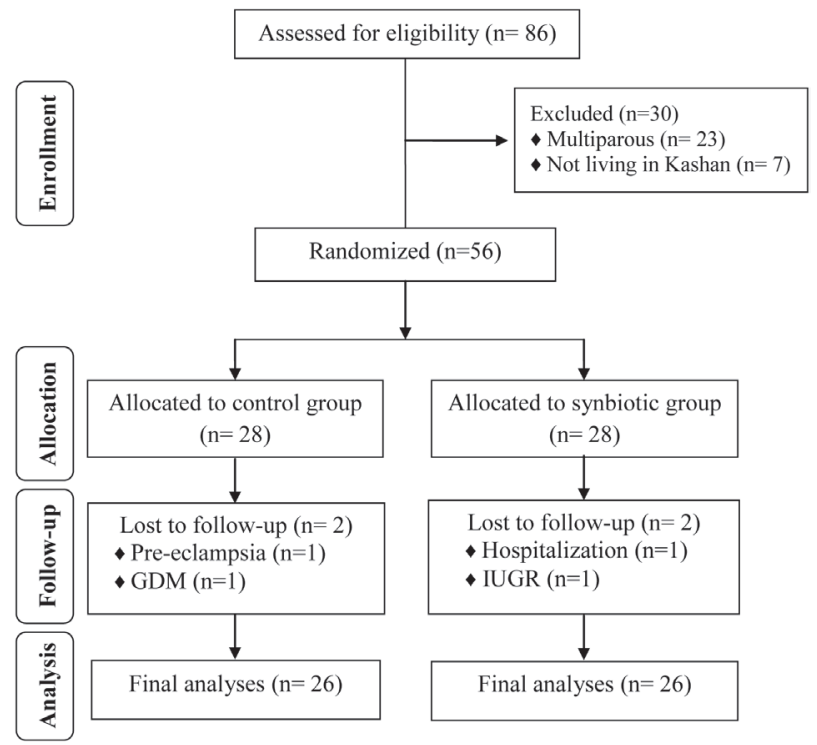

Figure 1. Summary of study participant flow. GDM: Gestational diabetes mellitus; IUGR: Intrauterine growth retardation.

\section{Study design}

To obtain detailed information about the dietary intakes of the study participants, all women participated in a 2-wk run-in period during which all subjects had to refrain from taking synbiotic or any other probiotic food. At the end of the run-in period (27 weeks of gestation), subjects were randomly assigned to consume $18 \mathrm{~g} / \mathrm{d}$ of synbiotic or control food for 9 weeks. The study was triple blind for the synbiotic and control-consuming groups. That is, in addition to the subjects and the investigator, the evaluator of the results was also not aware which treatment any particular subject received. Women who were pregnant were stratified based on age and BMI, then randomly assigned to the synbiotic or control group from computer generated random number lists. Randomization and allocation were concealed from the researcher and participants until after the main analyses had been completed. A trained midwife at the maternity clinic generated the randomized allocation sequence, enrolled participants and assigned participants to interventions. Participants were asked not to alter their routine physical activity or usual diets and not to consume any synbiotic or probiotic other than that provided to them by the investigators. They were also asked to avoid consuming any fermented products. Synbiotic or control foods were provided for the participants every week. Compliance with synbiotic or control food consumption was monitored once a week through phone interviews. The compliance was also double-checked by the use of three-day dietary records completed throughout the study. To obtain nutrient intakes of participants based on these three-day food diaries, we used Nutritionist IV software (First Databank, San Bruno, CA) modified for Iranian foods.

\section{Assessment of variables}

Data on pre-pregnancy weight and height (measured values) were taken from the records of the pregnant women who were in the clinic. A trained midwife at the maternity clinic took anthropometric measurements at study baseline and 9 weeks after intervention. Body weight was measured in an overnight fasting status, without shoes and in a minimal clothing state by the use of a digital scale (Seca, Hamburg, Germany) to the nearest $0.1 \mathrm{~kg}$. Height was measured using a non-stretched tape measure (Seca, Hamburg, Germany) to the nearest $0.1 \mathrm{~cm}$. BMI was calculated as weight in $\mathrm{kg}$ divided by height in meters squared.

\section{Biochemical assessment}

Fasting blood samples $(10 \mathrm{~mL})$ were taken at baseline and after 9-wk intervention at the Kashan reference laboratory in the early morning after an overnight fast. Fasting plasma glucose (FPG) levels were quantified by the use of glucose oxidase/peroxidase (GOD-POD) method with commercially available kits (Pars Azmun, Tehran, Iran). Serum insulin levels were assayed by enzyme linked immunoassay kits (DiaMetra, Milano, Italy). The intra- and interassay CVs for insulin were 3.1 and $6.2 \%$, respectively. The homeostatic model assessment for insulin resistance (HOMA-IR), $\beta$-cell function (HOMA-B) and the quantitative insulin sensitivity check index (QUICKI) was calculated based on suggested formulas. ${ }^{28}$ Serum hs-CRP concentration was assayed using ELISA kits (LDN, Nordhorn, Germany). The inter- and intraassay CVs for the hs-CRP assays ranged from 5.1 to $7.5 \%$. Measurements of FPG, insulin and hs-CRP were done in a blinded fashion, in duplicate, in pairs (before/after intervention) at the same time, in the same analytical run and in random order to reduce systematic error and inter-assay variability. 


\section{Synbiotic and control foods}

The synbiotic food consisted of a probiotic viable and heat-resistant Lactobacillus sporogenes (1X10 CFU), $0.04 \mathrm{~g}$ inulin (HPX) as prebiotic with $0.38 \mathrm{~g}$ isomalt, $0.36 \mathrm{~g}$ sorbitol and $0.05 \mathrm{~g}$ stevia as sweetener per $1 \mathrm{~g}$. The pregnant women were asked to consume the synbiotic food 2 times a day from a $9 \mathrm{~g}$ package. Therefore, they received $18 \times 10^{7} \mathrm{CFU}$ Lactobacillus sporogenes and $0.72 \mathrm{~g}$ inulin each day. Control food (the same substance without probiotic bacteria and prebiotic inulin) was packed in identical packages and coded by the producer to guarantee blinding. The synbiotic and control foods were provided by Sekkeh Gaz Company, Isfahan, Iran.

\section{Statistical analysis}

To ensure the normal distribution of variables, a histogram test was applied. For non-normally distributed variables, log-transformation was applied. Descriptive statistics (means and SDs) for general characteristics of the study participants were reported. Data on dietary intakes were compared by the paired t-test. The paired-samples $t$ test was used to detect within-group differences. The Student's t test was used to detect differences between the two groups (control and synbiotic foods). To determine the effect of synbiotic food on glycemic status and serum hs-CRP, we applied repeated measures analysis of variance where treatment*time interactions were tested by using Pillai's trace. In these analyses, the treatments (synbiotic and control foods) were regarded as between-subject factors and time with two time-points (baseline and week 9 of intervention) was considered as a within-subject factor. To assess whether the magnitude of the change depended on the starting value, we conditioned all analyses on baseline values to avoid the potential bias that might have resulted. All statistical analyses were done using the Statistical Package for Social Science version 17 (SPSS Inc., Chicago, Illinois, USA).

\section{RESULTS}

No serious adverse reactions were reported following consumption of the synbiotic food in the pregnant women throughout the study. We found neither a significant difference in the mean value of age, nor of pre-pregnancy weight and BMI between the two groups (Table 1). Baseline weight and BMI as well as their means after intervention were not significantly different between the control and synbiotic groups.

At the study beginning, no significant differences were found between the two groups in terms of dietary intakes. Comparing the dietary intakes during the run-in period and throughout the study separately in each group, we observed no significant within-group differences in dietary intakes except for monounsaturated fatty acids (MUFA) in the control group $(\mathrm{P}=0.013)$ and for dietary fiber in the symbiotic group $(\mathrm{P}=0.022)$ (Table 2). Based on the three-day dietary records throughout the study, no statistically significant difference was seen between the two groups in terms of dietary intakes of energy, carbohydrates, protein, fat, saturated fatty acids (SFA), polyunsaturated fatty acids (PUFA), MUFA, cholesterol and dietary fiber.

However, consumption of a synbiotic food did not show any significant impact on insulin actions in the synbiotic group; compared to the control food, it resulted in a significant decrease in serum insulin levels ( -0.26 vs. $6.34 \mu \mathrm{IU} / \mathrm{mL}, \mathrm{P}=0.014)$, HOMA-IR $(-0.13$ vs. $1.13, P=0.033)$, a significant difference in HOMA-B (5.30 vs. $34.22, \mathrm{P}=0.040)$ and a significant rise in QUICKI score ( 0.002 vs. $-0.02, \mathrm{P}=0.022)$ (Table 3). We did not find a significant effect of the synbiotic food consumption on FPG and serum hsCRP levels. Within-group differences in the control group demonstrated a significant increase in serum

Table 1. General characteristics of the study participants

\begin{tabular}{lccc}
\hline & $\begin{array}{c}\text { Control } \\
\text { food } \\
(\mathbf{n = 2 6})\end{array}$ & $\begin{array}{c}\text { Synbiotic } \\
\text { food } \\
(\mathbf{n = 2 6})\end{array}$ & $\boldsymbol{P}$ \\
\hline Maternal age $(\mathrm{y})$ & $29.0 \pm 4.6$ & $26.4 \pm 6.3$ & 0.097 \\
Height $(\mathrm{cm})$ & $160.0 \pm 6.1$ & $160.5 \pm 7.3$ & 0.805 \\
Pre-pregnancy weight $(\mathrm{kg})^{*}$ & $67.1 \pm 10.6$ & $64.8 \pm 13.3$ & 0.478 \\
Weight at study baseline $(\mathrm{kg})$ & $72.2 \pm 11.5$ & $71.9 \pm 14.2$ & 0.935 \\
Weight at end-of-trial $(\mathrm{kg})$ & $75.6 \pm 11.5$ & $75.6 \pm 13.5$ & 0.997 \\
Pre-pregnancy BMI $\left(\mathrm{kg} / \mathrm{m}^{2}\right)^{*}$ & $26.2 \pm 3.7$ & $25.1 \pm 4.8$ & 0.381 \\
BMI at study baseline $\left(\mathrm{kg} / \mathrm{m}^{2}\right)$ & $28.2 \pm 4.1$ & $27.9 \pm 5.1$ & 0.851 \\
BMI at end-of-trial $\left(\mathrm{kg} / \mathrm{m}^{2}\right)$ & $29.5 \pm 4.1$ & $29.4 \pm 4.9$ & 0.916 \\
\hline
\end{tabular}

Data are means \pm standard deviation. P-values were derived using independent $t$ test.

*Based on participants' measured weight and height as registered in their records in the maternity clinics. 
Table 2. Dietary intakes of study participants during the run-in period and throughout the study

\begin{tabular}{|c|c|c|c|c|c|c|c|}
\hline & \multicolumn{3}{|c|}{ Control food } & \multicolumn{3}{|c|}{ Synbiotic food } & \multirow[t]{2}{*}{$P^{* *}$} \\
\hline & $\begin{array}{l}\text { Run-in } \\
(n=26)\end{array}$ & $\begin{array}{c}\text { Throughout } \\
\text { the study }(n=26)\end{array}$ & $P^{*}$ & $\begin{array}{l}\text { Run-in } \\
(n=26)\end{array}$ & $\begin{array}{c}\text { Throughout } \\
\text { the study }(n=26)\end{array}$ & $P^{*}$ & \\
\hline Energy (kcal/d) & $2324 \pm 203$ & $2384 \pm 237$ & 0.542 & $2370 \pm 141$ & $2396 \pm 239$ & 0.368 & 0.659 \\
\hline Carbohydrates (g/d) & $326.8 \pm 30.2$ & $326.4 \pm 45.1$ & 0.955 & $323.2 \pm 48.9$ & $337.3 \pm 39.6$ & 0.315 & 0.363 \\
\hline Protein $(\mathrm{g} / \mathrm{d})$ & $88 \pm 9.5$ & $88 \pm 13.2$ & 0.991 & $85.1 \pm 18.9$ & $93.6 \pm 21.4$ & 0.099 & 0.143 \\
\hline Fat $(\mathrm{g} / \mathrm{d})$ & $82.5 \pm 12.5$ & $87.5 \pm 10.2$ & 0.118 & $79.9 \pm 18.1$ & $82.6 \pm 14.6$ & 0.642 & 0.715 \\
\hline SFA $(g / d)$ & $23.8 \pm 5.6$ & $26.3 \pm 3.8$ & 0.067 & $22.8 \pm 7.4$ & $24.1 \pm 5.6$ & 0.488 & 0.609 \\
\hline PUFA (g/d) & $27.6 \pm 6.4$ & $25.9 \pm 5.5$ & 0.279 & $27.6 \pm 6.7$ & $23.6 \pm 5$ & 0.067 & 0.372 \\
\hline $\operatorname{MUFA}(\mathrm{g} / \mathrm{d})$ & $21.7 \pm 5.1$ & $25.4 \pm 5.9$ & 0.013 & $20.9 \pm 7.5$ & $22.8 \pm 6.9$ & 0.407 & 0.504 \\
\hline Cholesterol (mg/d) & $210.8 \pm 110.8$ & $216.7 \pm 107.1$ & 0.815 & $209.3 \pm 159.1$ & $190.6 \pm 102.9$ & 0.504 & 0.512 \\
\hline Dietary fiber (g/d) & $18.1 \pm 4$ & $19.5 \pm 4.2$ & 0.180 & $17.9 \pm 4.8$ & $20.5 \pm 3.8$ & 0.022 & 0.397 \\
\hline
\end{tabular}

Data are means \pm standard deviations.

*P-values were obtained via the paired $t$ test. ${ }^{*} P$-values were obtained via the independent $t$ test for the comparison of dietary intakes throughout the study between the two groups.

SFA: Saturated fatty acid; PUFA: Polyunsaturated fatty acid; MUFA: Monounsaturated fatty acid.

Table 3. Means ( \pm standard deviation) of glycemic status and serum hs-CRP at baseline and after the intervention

\begin{tabular}{|c|c|c|c|c|c|c|c|c|c|}
\hline & \multicolumn{3}{|c|}{ Control food $(n=26)$} & \multicolumn{3}{|c|}{ Synbiotic food $(n=26)$} & \multicolumn{3}{|c|}{$P$} \\
\hline & Wk0 & Wk9 & Change & Wk0 & Wk9 & Change & Time & Group & $\begin{array}{l}\text { Time* } \\
\text { Group }\end{array}$ \\
\hline $\mathrm{FPG}(\mathrm{mg} / \mathrm{dL})$ & $72.80 \pm 10.36$ & $69.92 \pm 14.81$ & $-2.88 \pm 13.67$ & $65.26 \pm 22.93$ & $62.88 \pm 17.81$ & $-2.38 \pm 23.39$ & 0.325 & 0.070 & 0.925 \\
\hline Insulin, $\mu \mathrm{IU} / \mathrm{mL}$ & $9.40 \pm 7.89$ & $15.74 \pm 15.19^{*}$ & $6.34 \pm 9.83$ & $11.79 \pm 8.61$ & $11.53 \pm 6.56$ & $-0.26 \pm 8.72$ & 0.022 & 0.717 & 0.014 \\
\hline HOMA-IR & $1.63 \pm 1.29$ & $2.76 \pm 3.10^{*}$ & $1.13 \pm 2.27$ & $1.95 \pm 1.73$ & $1.82 \pm 1.32$ & $-0.13 \pm 1.86$ & 0.088 & 0.515 & 0.033 \\
\hline HOMA-B & $45.78 \pm 45.04$ & $80.00 \pm 76.16^{*}$ & $34.22 \pm 49.43$ & $63.72 \pm 41.76$ & $69.02 \pm 51.92$ & $5.30 \pm 49.31$ & 0.006 & 0.801 & 0.040 \\
\hline QUICKI & $0.37 \pm 0.04$ & $0.35 \pm 0.05^{*}$ & $-0.02 \pm 0.04$ & $0.36 \pm 0.03$ & $0.36 \pm 0.03$ & $0.002 \pm 0.03$ & 0.038 & 0.908 & 0.022 \\
\hline Hs-CRP, ng/mL & $6733.0 \pm 4078.2$ & $5664.7 \pm 3652.8$ & $-1068.3 \pm 4148.1$ & $5041.2 \pm 4053.3$ & $4563.2 \pm 4124.6$ & $-478.0 \pm 1581.6$ & 0.082 & 0.175 & 0.501 \\
\hline
\end{tabular}

$P$-values obtained from repeated measures ANOVA test.

FPG: Fasting plasma glucose; HOMA-IR: Homeostasis model of assessment-insulin resistance; HOMA-B: Homeostatic model assessment-Beta cell function; QUICKI: Quantitative insulin sensitivity check index; Hs-CRP: High sensitivity C-reactive protein. *Different from wk $0, \mathrm{P}<0.05$.

insulin levels $(6.34 \mu \mathrm{IU} / \mathrm{mL}, \mathrm{P}=0.003)$, HOMA-IR $(1.13, \mathrm{P}=0.017)$, HOMA-B $(34.22, \mathrm{P}=0.002)$ and a significant decrease in QUICKI score $(-0.02, \mathrm{P}=0.006)$.

When the analyses were adjusted for baseline values, no significant changes in our findings were observed (Table 4). Furthermore, control for maternal age did not alter in our findings except for HOMA-IR ( $\mathrm{P}=0.052)$.

\section{DISCUSSION}

Our study revealed that the intake of synbiotic food for 9 weeks among pregnant women in the third trimester resulted in a significant reduction of serum insulin levels, HOMA-IR, HOMA-B and a significant elevation of QUICKI score compared to the control food, but did not affect FPG and serum hs-CRP levels. However, earlier studies on the effects of synbiotics have mostly been assessed in vitro ${ }^{29}$ and patients with multiple injuries; ${ }^{30}$ thus, to the best of our knowledge, this study is the first examining the effects in pregnant women.

Pregnant women are very susceptible to insulin resistance and increased inflammatory factors, es- 
Table 4. Adjusted changes in glycemic status and serum hs-CRP in pregnant women who received either synbiotic or control foods

\begin{tabular}{cccc}
\hline & $\begin{array}{c}\text { Control food } \\
(\mathbf{n = 2 6})\end{array}$ & $\begin{array}{c}\text { Synbiotic food } \\
(\mathbf{n = 2 6})\end{array}$ & $\mathbf{P}$ \\
\hline FPG (mg/dL) & & & \\
Model 1* & $-0.41 \pm 3.04$ & $-4.85 \pm 3.04$ & 0.313 \\
Model 2** & $-3.60 \pm 3.80$ & $-1.66 \pm 3.80$ & 0.724 \\
Insulin, $\mu$ IU/mL & & & \\
Model 1 & $6.18 \pm 1.83$ & $-0.09 \pm 1.83$ & 0.020 \\
Model 2 & $6.39 \pm 1.86$ & $-0.30 \pm 1.86$ & 0.016 \\
HOMA-IR & & & \\
Model 1 & $1.10 \pm 0.40$ & $-0.09 \pm 0.40$ & 0.044 \\
Model 2 & $1.09 \pm 0.41$ & $-0.09 \pm 0.41$ & 0.052 \\
HOMA-B & & & \\
Model 1 & $34.04 \pm 9.88$ & $5.47 \pm 9.88$ & 0.049 \\
Model 2 & $36.26 \pm 9.76$ & $3.25 \pm 9.76$ & 0.022 \\
QUICKI & & & \\
Model 1 & $-0.020 \pm 0.007$ & $0.001 \pm 0.007$ & 0.037 \\
Model 2 & $-0.020 \pm 0.007$ & $0.004 \pm 0.007$ & 0.011 \\
Hs-CRP, ng/mL & & & \\
Model 1 & $-781.2 \pm 565.0$ & $-764.2 \pm 565.0$ & 0.983 \\
Model 2 & $-107.3 \pm 630.7$ & $-474.2 \pm 630.7$ & 0.512 \\
\hline P-Values 0btained & & & \\
\hline
\end{tabular}

$P$-values obtained from ANCOVA.

FPG: Fasting plasma glucose; HOMA-IR: Homeostasis model of assessment-insulin resistance; HOMA-B: Homeostatic model assessment-Beta cell function; QUICKI: Quantitative insulin sensitivity check index; Hs-CRP: High sensitivity C-reactive protein. *Adjusted for baseline values (data are means \pm standard error), **Adjusted for maternal age (data are means \pm standard error).

pecially in the third trimester. Insulin resistance and elevated inflammatory factors during pregnancy can result in several complications in mother and fetus. ${ }^{9,10}$ We revealed that supplementation of the synbiotic food significantly decreased serum insulin levels, HOMA-IR and HOMA-B and increased QUICKI score compared to the control food, but did not affect FPG levels compared with the control food. The beneficial effects of synbiotics and probiotics on serum insulin levels and insulin resistance have previously been reported. Our previous study among diabetic patients showed that consumption of a synbiotic food containing Lactobacillus sporogenes $\left(27 \times 10^{7} \mathrm{CFU}\right)$ and $1.08 \mathrm{~g}$ inulin results in a significant decrease in serum insulin levels after 6 weeks compared with the control food, but did not affect HOMA-IR score. ${ }^{21}$ Supplementation of Lacto- bacillus acidophilus for 4 weeks among type 2 diabetic patients also preserved insulin sensitivity compared with the placebo group. ${ }^{31}$ Improved glucose tolerance and insulin resistance has also been observed following consumption of several strains of bacteria, such as Lactobacilli and Bifidobacterium in animal models. ${ }^{32-35}$ The administration of Lactobacillus reuteri in high fructose-fed rats for 12 weeks significantly suppressed the elevation of serum glucose and insulin levels as well as improving insulin resistance. ${ }^{36}$ Improved glucose tolerance and glucose-induced insulin secretion was also seen with the consumption of Bifidobacterium in diabetic mice fed a high-fat diet. ${ }^{33}$ Besides individual probiotics, a combination of their strains has also been suggested as being advantageous in reducing the onset of insulin resistance and diabetes in animal models. Intake of probiotics VSL\#3 containing Bifidobacteria, Lactobacilli and Streptococcus thermophilus for 4 weeks in diabetic mice improved hepatic insulin resistance. ${ }^{37}$ The same findings have also been registered by consumption of Bifidobacterium breve B-3 at $10^{8}$ or $10^{9} \mathrm{CFU} / \mathrm{d}$ for 8 weeks in mice fed a high-fat $\operatorname{diet}^{35}$ and Lactobacillus casei $0.05 \%$ for 4 weeks in mice. ${ }^{38}$ As is clear from the abovementioned studies, most studies have been done on animal models and limited data are available among humans. However, the consumption of probiotic supplements did not affect FPG, serum insulin levels and HOMA-IR among patients with T2D after 8 weeks compared with the placebo. ${ }^{39}$ Several mechanisms possibly account for the beneficial effects of synbiotic food on serum insulin levels and insulin resistance. The effects on insulin sensitivity might be attributed to their impact on gene expression that results in adiposity. ${ }^{40}$ Furthermore, SCFA production, especially butyrate, by probiotics promoted the release of the hormone glucagon-like peptide-1 (GLP-1) from intestinal L-cells resulting in improved glucose tolerance. ${ }^{25}$ In addition, the effect of synbiotics on changes in levels of gut hormones like peptide YY (PYY), ${ }^{41}$ activation of lipopolysaccharide toll-like receptor- $2^{42}$ and changes in the intestinal barrier integrity ${ }^{43}$ might provide some reasons for their effects on circulating insulin levels and glycemic status.

We demonstrated that the synbiotic food consumption does not affect serum hs-CRP levels. In line with our study, intake of synbiotic did not promote any significant changes in inflammatory cytokines in 
healthy elderly individuals after 3 months. ${ }^{23}$ Supplementation of probiotic did not show any significant difference in serum hs-CRP levels among diabetic patients after 6 weeks compared with the placebo. ${ }^{44}$ The same findings were recorded with consumption of Lactobacillus rhamnosus GG among patients with rheumatoid arthritis ${ }^{45}$ and the use of Lactobacillus plantarum in critically ill patients. ${ }^{46}$ However, intake of a synbiotic food containing Lactobacillus casei, Bifidobacterium breve and galacto-oligosaccharides resulted in serum hs-CRP levels among patients undergoing hepatobiliary resection. ${ }^{47}$ Similar findings have also been observed with consumption of a synbiotic food in patients with severe multiple injuries for 7 and 15 days ${ }^{30}$ and a synbiotic containing Bifidobacterium, Lactobacillus and galacto-oligosaccharides among hepatectomized patients with or without liver cirrhosis. ${ }^{48}$ The absence of any effect in our own study of synbiotic food consumption on serum hs-CRP levels may result from the fact that our study design, patients under investigation as well as duration of supplementation differed from those of other studies. Several limitations must be considered in the interpretation of our findings. First of all, due to budget limitations we unable to assess favorable effects of the synbiotic food on other inflammatory markers including IL-1, IL-6 and tumor necrosis factor alpha (TNF- $\alpha$ ). Secondly, we could not assess the effects of synbiotic-containing food on pregnancy outcomes.

\section{CONCLUSIONS}

In conclusion, consumption of a synbiotic food for 9 weeks by pregnant women had beneficial effects on insulin actions compared to the control food, but did not affect FPG and serum hs-CRP concentrations.

\section{ACKNOWLEDGEMENTS}

The present study was supported by a grant (no. 9013) from the Vice-Chancellor for Research, KUMS, Kashan, Iran. The authors would like to thank the staff of the Naghavi and Shaheed Beheshti Gynecology Clinics (Kashan, Iran) for their assistance in this project. We are grateful to the Research and Development Division of Sekkeh Gaz Company, Isfahan, Iran, that provided the synbiotic product for the present study.

\section{CONFLICT OF INTEREST}

None of the authors had any personal or financial conflict of interest.

Clinical trial registration number: http://www.irct.ir. IRCT201212105623N3.

\section{REFERENCES}

1. Ensling M, Steinmann W, Whaley-Connell A, 2011 Hypoglycemia: A Possible Link between Insulin Resistance, Metabolic Dyslipidemia, and Heart and Kidney Disease (the Cardiorenal Syndrome). Cardiorenal Med 1: 67-74.

2. Johnson CA, 2008 Glucose homeostasis during canine pregnancy: Insulin resistance, ketosis, and hypoglycemia. Theriogenology 70: 1418-1423.

3. McLachlan KA, O'Neal D, Jenkins A, Alford FP, 2006 Do adiponectin, TNFalpha, leptin and CRP relate to insulin resistance in pregnancy? Studies in women with and without gestational diabetes, during and after pregnancy. Diabetes Metab Res Rev 22: 131-138.

4. Lu HL, Wang HW, Wen Y, Zhang MX, Lin HH, 2006 Roles of adipocyte derived hormone adiponectin and resistin in insulin resistance of type 2 diabetes. World J Gastroenterol 12: 1747-1751.

5. Senn JJ, Klover PJ, Nowak IA, Mooney RA, 2002 Interleukin-6 induces cellular insulin resistance in hepatocytes. Diabetes 51: 3391-3399.

6. Briana DD, Malamitsi-Puchner A, 2009 Reviews: adipocytokines in normal and complicated pregnancies. Reprod Sci 16: 921-937.

7. Clapp JF, 2006 Effects of Diet and Exercise on Insulin Resistance during Pregnancy. Metab Syndr Relat Disord 4: 84-90.

8. Pitiphat W, Gillman MW, Joshipura KJ, Williams PL, Douglass CW, Rich-Edwards JW, 2005 Plasma Creactive protein in early pregnancy and preterm delivery. Am J Epidemiol 162: 1108-1113.

9. Stella CL, O'Brien JM, Forrester KJ, et al, 2008 The coexistence of gestational hypertension and diabetes: influence on pregnancy outcome. Am J Perinatol 25: 325-329.

10. Kayemba-Kay's S, Peters C, Geary MP, Hill NR, Mathews DR, Hindmarsh PC, 2013 Maternal hyperinsulinism and glycaemic status in the first trimester of pregnancy are associated with the development of pregnancy-induced hypertension and gestational diabetes. Eur J Endocrinol 168: 413-418.

11. Karcaaltincaba D, Kandemir O, Yalvac S, GuvendagGuven S, Haberal A, 2009 Prevalence of gestational diabetes mellitus and gestational impaired glucose tolerance in pregnant women evaluated by National Diabetes Data Group and Carpenter and Coustan criteria. 
Int J Gynaecol Obstet 106: 246-249.

12. Cheung NW, 2009 The management of gestational diabetes. Vasc Health Risk Manag 5: 153-164.

13. Seshiah V, Balaji V, Balaji MS, et al, 2007 Gestational diabetes mellitus manifests in all trimesters of pregnancy. Diabetes Res Clin Pract 77: 482-484.

14. Katon J, Maynard C, Reiber G, 2012 Attempts at weight loss in U.S. women with and without a history of gestational diabetes mellitus. Women's Health Issues 22: e447-453.

15. Ferrara A, Hedderson MM, Albright CL, et al, 2011 A Pregnancy and Postpartum Lifestyle Intervention in Women With Gestational Diabetes Mellitus Reduces Diabetes Risk Factors: A feasibility randomized control trial. Diabetes Care 34: 1519-1525.

16. Prater MR, Laudermilch CL, Holladay SD, 2007 Does immune stimulation or antioxidant therapy reduce MNU-induced placental damage via activation of JakSTAT and NFkappaB signaling pathways? Placenta 28: 566-570.

17. Lawrence JM, Andrade SE, Avalos LA, et al, 2013 Prevalence, trends, and patterns of use of antidiabetic medications among pregnant women, 2001-2007. Obstet Gynecol 121: 106-114.

18. Borzychowski AM, Sargent IL, Redman CW, 2006 Inflammation and pre-eclampsia. Semin Fetal Neonatal Med 11: 309-316.

19. Asemi Z, Samimi M, Tabassi Z, et al, 2013 Effect of daily consumption of probiotic yoghurt on insulin resistance in pregnant women: a randomized controlled trial. Eur J Clin Nutr 67: 71-74.

20. Asemi Z, Jazayeri S, Najafi M, et al, 2011 Effects of daily consumption of probiotic yoghurt on inflammatory factors in pregnant women: a randomized controlled trial. Pak J Biol Sci 14: 476-482.

21. Asemi Z, Khorrami-Rad A, Alizadeh SA, Shakeri H, Esmaillzadeh A, 2013 Effects of synbiotic food consumption on metabolic status of diabetic patients: a double-blind randomized cross-over controlled clinical trial. Clin Nutr 33: 198-203.

22. D'Souza A, Fordjour L, Ahmad A, et al, 2010 Effects of probiotics, prebiotics, and synbiotics on messenger RNA expression of caveolin-1, NOS, and genes regulating oxidative stress in the terminal ileum of formula-fed neonatal rats. Pediatr Res 67: 526-531.

23. Neto JV, de Melo CM, Ribeiro SM, 2013 Effects of three-month intake of synbiotic on inflammation and body composition in the elderly: a pilot study. Nutrients 5: 1276-1286.

24. Vitali B, Ndagijimana M, Cruciani F, et al, 2010 Impact of a synbiotic food on the gut microbial ecology and metabolic profiles. BMC Microbiol 10: 4.

25. Yadav H, Lee JH, Lloyd J, Walter P, Rane SG, 2013 Beneficial metabolic effects of a probiotic via butyrate induced GLP-1 secretion. J Biol Chem 288: 2508825097.
26. Voltolini C, Battersby S, Etherington SL, Petraglia F, Norman JE, Jabbour HN, 2012 A novel antiinflammatory role for the short-chain fatty acids in human labor. Endocrinology 153: 395-403.

27. Jehan I, Zaidi S, Rizvi S, et al, 2010 Dating gestational age by last menstrual period, symphysis-fundal height, and ultrasound in urban Pakistan. Int J Gynaecol Obstet 110: 231-234.

28. Pisprasert V, Ingram KH, Lopez-Davila MF, Munoz AJ, Garvey WT, 2013 Limitations in the use of indices using glucose and insulin levels to predict insulin sensitivity: impact of race and gender and superiority of the indices derived from oral glucose tolerance test in African Americans. Diabetes Care 36: 845-853.

29. Vitali B, Ndagijimana M, Maccaferri S, Biagi E, Guerzoni ME, Brigidi P, 2012 An in vitro evaluation of the effect of probiotics and prebiotics on the metabolic profile of human microbiota. Anaerobe 18: 386-391.

30. Giamarellos-Bourboulis EJ, Bengmark S, Kanellakopoulou K, Kotzampassi K, 2009 Pro- and synbiotics to control inflammation and infection in patients with multiple injuries. J Trauma 67: 815-821.

31. Andreasen AS, Larsen N, Pedersen-Skovsgaard T, et al, 2010 Effects of Lactobacillus acidophilus NCFM on insulin sensitivity and the systemic inflammatory response in human subjects. Br J Nutr 104: 18311838 .

32. Tabuchi M, Ozaki M, Tamura A, et al, 2003 Antidiabetic effect of Lactobacillus GG in streptozotocin-induced diabetic rats. Biosci Biotechnol Biochem 67: 1421-1424.

33. Cani PD, Neyrinck AM, Fava F, et al, 2007 Selective increases of bifidobacteria in gut microflora improve high-fat-diet-induced diabetes in mice through a mechanism associated with endotoxaemia. Diabetologia 50: 2374-2383.

34. Musso G, Gambino R, Cassader M, 2010 Obesity, diabetes, and gut microbiota: the hygiene hypothesis expanded? Diabetes Care 33: 2277-2284.

35. Kondo S, Xiao JZ, Satoh T, et al, 2010 Antiobesity effects of Bifidobacterium breve strain B-3 supplementation in a mouse model with high-fat diet-induced obesity. Biosci Biotechnol Biochem 74: 1656-1661.

36. Hsieh FC, Lee CL, Chai CY, Chen WT, Lu YC, Wu CS, 2013 Oral administration of Lactobacillus reuteri GMNL-263 improves insulin resistance and ameliorates hepatic steatosis in high fructose-fed rats. Nutr Metab (Lond) 10: 35.

37. Li Z, Yang S, Lin H, et al, 2003 Probiotics and antibodies to TNF inhibit inflammatory activity and improve nonalcoholic fatty liver disease. Hepatology 37: 343350 .

38. Naito E, Yoshida Y, Makino K, et al, 2011 Beneficial effect of oral administration of Lactobacillus casei strain Shirota on insulin resistance in diet-induced obesity mice. J Appl Microbiol 110: 650-657.

39. Asemi Z, Zare Z, Shakeri H, Sabihi S-S, Esmaillzadeh 
A, 2013 Effect of multispecies probiotic supplements on metabolic profile, hs-CRP and oxidative stress in patients with type 2 diabetes. Ann Nutr Metab 63: 1-9.

40. Esteve E, Ricart W, Fernandez-Real JM, 2011 Gut microbiota interactions with obesity, insulin resistance and type 2 diabetes: did gut microbiote co-evolve with insulin resistance? Curr Opin Clin Nutr Metab Care 14: 483-490.

41. Diamant M, Blaak EE, de Vos WM, 2011 Do nutrientgut-microbiota interactions play a role in human obesity, insulin resistance and type 2 diabetes? Obes Rev 12: 272-281.

42. Caricilli AM, Picardi PK, de Abreu LL, et al, 2011 Gut microbiota is a key modulator of insulin resistance in TLR 2 knockout mice. PLoS Biol 9: e1001212.

43. Cani PD, Possemiers S, Van de Wiele T, et al, 2009 Changes in gut microbiota control inflammation in obese mice through a mechanism involving GLP-2-driven improvement of gut permeability. Gut 58: 1091-1103.

44. Mazloom Z, Yousefinejad A, Dabbaghmanesh MH,
2013 Effect of probiotics on lipid profile, glycemic control, insulin action, oxidative stress, and inflammatory markers in patients with type 2 diabetes: a clinical trial. Iran J Med Sci 38: 38-43.

45. Hatakka K, Martio J, Korpela M, et al, 2003 Effects of probiotic therapy on the activity and activation of mild rheumatoid arthritis--a pilot study. Scand J Rheumatol 32: 211-215.

46. McNaught CE, Woodcock NP, Anderson AD, MacFie $\mathrm{J}, 2005$ A prospective randomised trial of probiotics in critically ill patients. Clin Nutr 24: 211-219.

47. Sugawara G, Nagino M, Nishio H, et al, 2006 Perioperative synbiotic treatment to prevent postoperative infectious complications in biliary cancer surgery: a randomized controlled trial. Ann Surg 244: 706-714.

48. Usami M, Miyoshi M, Kanbara Y, et al, 2011 Effects of perioperative synbiotic treatment on infectious complications, intestinal integrity, and fecal flora and organic acids in hepatic surgery with or without cirrhosis. JPEN J Parenter Enteral Nutr 35: 317-328. 